\title{
Determinants of bone diseases in tenofovir-treated HIV patients
}

Cristina Gervasoni ${ }^{1}$, Davide Minisci ${ }^{1}$, Paola Meraviglia ${ }^{1}$, Sara Baldelli ${ }^{2}$, Emilio Clementi ${ }^{3,4}$, Giuliano Rizzardini ${ }^{1,5}$, Dario Cattaneo ${ }^{2}$

${ }^{1}$ Department of Infectious Diseases, L. Sacco University Hospital, Milan, Italy

${ }^{2}$ Unit of Clinical Pharmacology, L. Sacco University Hospital, Milan, Italy

${ }^{3}$ Clinical Pharmacology Unit, CNR Institute of Neuroscience, Dept Biomedical and

Clinical Sciences, L. Sacco University Hospital, Università di Milano, Milan, Italy

${ }^{4}$ Scientific Institute IRCCS E.Medea, 23842 Bosisio Parini, Italy

${ }^{5}$ School of Clinical Medicine, University of the Witwatersrand, Johannesburg

Correspondence to: Cristina Gervasoni, M.D.

$3^{\text {rd }}$ Division of Infectious Diseases

Luigi Sacco University Hospital

Via GB Grassi 74, 20157 Milano, Italy

E mail: cristina.gervasoni@unimi.it 
Bedimo and Co-workers have recently reported the results of a prospective, crosssectional study aimed at investigating the mechanisms of increased bone loss in male subjects with HIV and HCV mono-infections or HIV/HCV co-infections [1]. They found that HIV and HCV were significantly and independently associated with lower bone mineral density (BMD). The statistical models adjusted for tenofovir (TDF) use greatly attenuated the association of HIV with decreased BMD, suggesting that the effect of HIV on BMD was mainly driven by TDF. Furthermore, the Authors documented that, at variance with HCV, HIV infection was associated also with altered bone turnover, as documented by increased telopeptide X (CTX) and osteocalcin (OC) serum concentrations. Here we intend to extend these findings by assessing the potential contribution of TDF overexposure on bone diseases, in consideration of the recently reported associations between high TDF plasma concentrations and the development of kidney impairment [2,3].

In this retrospective analysis we included all HIV-infected patients from our local database treated with TDF for at least six months and with at least one assessment of TDF plasma trough concentrations. Bone diseases were assessed through evaluation of BMD by dual-energy x-ray absorptiometry (DXA) (osteopenia and osteoporosis were diagnosed as a spine or hip T-score between -1 and -2.5 or $\leq 2.5$, respectively) and/or by markers of bone formation and resorption such as OC (reference values: 11-46 ng/mL) and CTX (reference values: $<580,<704$ and $<1000 \mathrm{pg} / \mathrm{mL}$ for all patients $<50$ years, males $>50$ years and females $>50$ years, respectively). 198 out of the 478 patients from our database fulfilling the inclusion criteria performed at least one DXA evaluation. They were at a mean of $2080 \pm 1209$ days of therapy with TDF and were mainly given a 
protease inhibitor (54\%) or a non-nucleoside reverse transcriptase inhibitor (34\%) as the third drug of the antiretroviral therapy. Overall, 150 out of the 198 patients (76\%) had DXA alterations (92 and 90 patients with osteopenia and osteoporosis, respectively). As shown in Table 1, these patients had lower body mass index, were older and were treated longer with TDF as compared to patients with normal DXA, whereas no differences on TDF trough concentrations were found $(157 \pm 139$ vs. $142 \pm 136 \mathrm{ng} / \mathrm{mL}$, $\mathrm{p}=0.51$ ). The same trends were confirmed also when repeating the analysis by considering only patients with osteoporosis. Data on markers of bone turnover were available for 133 out of the 478 patients. Significantly higher TDF concentrations were found in patients with altered vs. normal OC levels (TDF concentrations: $288 \pm 173$ vs. $153 \pm 115 \mathrm{ng} / \mathrm{mL}, \mathrm{p}<0.01)$. Conversely, no association between TDF concentrations and CTX level were documented (TDF concentrations: $147 \pm 103$ vs. $165 \pm 128 \mathrm{ng} / \mathrm{mL}$, $\mathrm{p}=0.74$ in patients with altered vs. normal CTX values).

In agreement with previous findings, we firstly documented that a large proportion of HIV-infected patients on long-term antiretroviral therapy developed bone metabolism alterations; these events were significantly associated with patients' age and lower body mass index [4,5]. We also extended the findings by Bedimo et al [1] by recognizing that bone disease was significantly associated with the duration of TDF therapy but not with TDF plasma trough concentrations. The latter finding seems to argue against a potential contribution of TDF overexposure on the development of bone diseases, at variance with findings in HIV-infected patients experiencing TDF-related kidney complications $[2,3]$. This may be eventually related to the fact that, at variance with kidney diseases in which a direct mechanism linked to the selective accumulation of TDF within renal 
tubular cells has been identified [6-8] - TDF is only one of the risk factors for bone loss, and its effect may be eventually diluted by the presence of other clinical covariates (such as, patients' sex, age, diet, body mass index, etc) known to play a key pathogenetic role in bone diseases [4,5]. This hypothesis has been indirectly confirmed by the findings that patients with altered OCT levels have significantly higher TDF plasma trough concentrations compared with patients with physiologic OC levels, whereas no association was found with CTX levels. Taken together, these results suggest an essential involvement of TDF only in the process of bone formation. Indeed, assuming that $\mathrm{OC}$ is a marker which a) is secreted solely by osteoblasts, b) is proosteoblastic and c) whose alterations induce pathologic alterations in BMD [9], it can be reasonably speculated that the detrimental effects of TDF on bone metabolism might be driven mainly by OC-mediated loss of osteoblast function. Accordingly, our results provide for the first time in vivo indirect evidence that the effect of TDF on osteoblast activity is concentration-dependent, as previously documented in vitro in osteoblast cultures [10].

In conclusion, in this hypothesis-generating study we have provided preliminary evidence that exposure of patients to high plasma TDF concentrations might induce early pathologic alterations in bone turnover, eventually favoring the development of osteopenia/osteoporosis through impaired bone formation in high-risk HIV-infected patients. 


\section{Disclosure statement}

DC has received educational/travel grants from Merck Sharp \& Dome, Bristol Myers Squibb, ViiV Healthcare and Janssen-Cilag.

CG and GR have received educational grants from Merck Sharp \& Dome, JanssenCilag, Bristol Myers Squibb, Boehringer Ingelheim and Abbvie

All Authors have read and approved the text. 


\section{References}

1. Bedimo R, Cutrell J, Zhang S, Drechsler H, Gao A, Brown G, et al. Mechanisms of bone disease in HIV and hepatitis C virus: impact of bone turnover, tenofovir exposure, sex steroids and severity of liver disease. AIDS. 2016;30:601-8

2. Rodríguez-Nóvoa S, Labarga P, D'avolio A, Barreiro P, Albalate M, Vispo E, et al. Impairment in kidney tubular function in patients receiving tenofovir is associated with higher tenofovir plasma concentrations. AIDS. 2010;24:1064-6.

3. Gervasoni C, Meraviglia P, Landonio S, Baldelli S, Fucile S, Castagnoli L, et al. Low body weight in females is a risk factor for increased tenofovir exposure and drug-related adverse events. PLoS One. 2013;8:e80242.

4. Pinnetti C, Federico L, Lorenzini P, Domenico C, Rita B, Laura L, et al. Relationship between body mass index and bone mineral density in HIVinfected patients referred for DXA. J Int AIDS Soc. 2014;17(4 Suppl 3):19569.

5. Warriner AH, Mugavero M, Overton ET. Bone alterations associated with HIV. Curr HIV/AIDS Rep. 2014;11:233-40.

6. Moss DM, Neary M, Owen A. The role of drug transporters in the kidney: lessons from tenofovir. Front Pharmacol. 2014;5:248. 
7. Hall AM. Update on tenofovir toxicity in the kidney. Pediatr Nephrol. 2013;28:1011-23.

8. Rodriguez-Nóvoa S, Alvarez E, Labarga P, Soriano V. Renal toxicity associated with tenofovir use. Expert Opin Drug Saf. 2010;9:545-59.

9. Zoch ML, Clemens TL, Riddle RC. New insights into the biology of osteocalcin. Bone. 2016;82:42-9.

10. Grigsby IF, Pham L, Mansky LM, Gopalakrishnan R, Carlson AE, Mansky KC. Tenofovir treatment of primary osteoblasts alters gene expression profiles: implications for bone mineral density loss. Biochem Biophys Res Commun. 2010;394:48-53. 
Table 1. Patients characteristics clustered according to the development or not of bone diseases

\begin{tabular}{llll}
\hline Parameters & $\begin{array}{l}\text { No bone } \\
\text { diseases } \\
(\mathbf{n = 4 8})\end{array}$ & $\begin{array}{l}\text { Osteopenia or } \\
\text { osteoporosis } \\
(\mathbf{n = 1 5 0 )}\end{array}$ & P-value \\
\hline TDF therapy, days & $1683 \pm 1417$ & $2209 \pm 1109$ & $<0.01$ \\
TDF concentrations, ng/mL & $142 \pm 136$ & $157 \pm 139$ & 0.51 \\
Female gender, \% & $47.9 \%$ & $43.3 \%$ & 0.33 \\
Age, years & $46 \pm 10$ & $51 \pm 8$ & $<0.01$ \\
Concomitant ARV drugs, \% & $50 \%$ PI & $55 \%$ PI & 0.74 \\
& $33 \%$ NNRTI & $35 \%$ NNRTI & \\
& $17 \%$ other & $10 \%$ other & \\
Body mass index, Kg/m ${ }^{2}$ & $23.8 \pm 4.6$ & $22.2 \pm 3.9$ & $<0.05$ \\
S.creatinine before TDF, mg/dL & $0.80 \pm 0.19$ & $0.78 \pm 0.19$ & 0.65 \\
S. creatinine last f.u., mg/dL & $1.0 \pm 0.3$ & $0.9 \pm 0.3$ & 0.16 \\
CD4, cells/mL & $585 \pm 252$ & $654 \pm 286$ & 0.15 \\
HBV or HCV coinfection, \% & $38 \%$ & $43 \%$ & 0.51 \\
\hline TDF: tenofovir; ARV: antiretroviral; PI: protease inhibitor; NNRTI: non-nucleoside reverse transcriptase inhibitor; \\
f.u.: follow-up; HBV: hepatitis B virus; HCV: hepatitis C virus & & \\
& & &
\end{tabular}

